\title{
Differential Modulation for Two-User Cooperative Diversity Systems
}

\author{
Poramate Tarasak, Member, IEEE, Hlaing Minn, Member, IEEE, and Vijay K. Bhargava, Fellow, IEEE
}

\begin{abstract}
This paper introduces a novel differential modulation scheme for a two-user cooperative diversity system which does not require channel state information at either the users or the destination. The performance of fixed decode-and-forward and selection relaying protocols is evaluated in both symmetric and asymmetric interuser-channel cases. The lower bound on the performance of the decode-and-forward protocol is given, while the exact bit-error probability of the selection relaying protocol is thoroughly derived. The decode-and-forward relaying protocol achieves a performance gain when the signal-to-noise ratios in the interuser channels are symmetric and sufficiently high. The selection relaying protocol shows a larger performance gain and does not exhibit an error floor like in the case of the decode-and-forward protocol. In addition, it is robust to the asymmetric interuser channels.
\end{abstract}

Index Terms-Asymmetric interuser channels, cooperative diversity, decode-and-forward relaying, differential modulation, selection relaying.

\section{INTRODUCTION}

$\mathbf{T}$ RADITIONAL space-time coding and multiple-input multiple-output (MIMO) techniques exploit spatial diversity through multiple transmit and/or receive antennas. However, due to the size of a mobile and carrier frequency constraints, achieving spatial diversity through multiple antennas may not be possible. Cooperative diversity has recently emerged as an alternative way to achieve diversity with a single antenna at the mobile. As there are many users in the wireless system, cooperative diversity allows sharing the resources (e.g., time, frequency) among cooperative users and each user can serve as a relay for the transmissions of other users' information. Diversity is obtained from the relay transmissions by other users in the system.

The concept of cooperative diversity was introduced in [3], where the capacity region, outage probabilities, coverage area were derived and code-division multiple-access (CDMA) implementation was discussed. In [4], outage probabilities for

Manuscript received April 1, 2004; revised November, 24, 2004, February 2, 2005, and February 20, 2005. This research was supported in part by the Natural Sciences and Engineering Research Council of Canada under a Strategic Project Grant. This paper was presented in part at GLOBECOM 2004, November 29-December 3, 2004, Dallas, TX.

P. Tarasak was with the Department of Electrical and Computer Engineering, University of Victoria, Victoria, BC V8W3P6, Canada. He is now with the Department of Electrical Engineering, Korea Advanced Institute of Science and Technology, Daejeon 373-1, Korea (e-mail: ptarasak@ece.uvic.ca; ptarasak@stein.kaist.ac.kr).

H. Minn is with the Department of Electrical Engineering, University of Texas at Dallas, Richardson, TX 75083-0688 USA (e-mail: hlaing.minn@ utdallas.edu).

V. K. Bhargava is with the Department of Electrical and Computer Engineering, University of British Columbia, Vancouver, BC V6T1Z4, Canada.

Digital Object Identifier 10.1109/JSAC.2005.853792 several relaying protocols were derived. It was concluded that except for the fixed decode-and-forward relaying protocol, all other protocols which are amplify-and forward relaying, selection relaying and incremental relaying achieve full diversity. In [5], an improved multiple-access scheme over [4] referred to as space-time coded protocol was proposed and it was shown to offer full spatial diversity with better bandwidth efficiency.

Previously mentioned works consider simple repetition codes and hard decisions at the relay mobiles or use a random coding argument to develop some performance bounds. The concept of coded cooperation, where the relay mobile is allowed to perform channel coding, was introduced in [6] and later extended in [7], where the ideas of space-time coding and turbo coding were exploited. Other related works include [8], where Alamouti's space-time block code [1] was applied in a wireless relay system [9], where a linear space-time block code was applied in a cooperative relay system in purely line-of-sight channels, and [10] where space-time signal design criteria were given for fading relay channels and the selection relaying protocol based on a cutoff rate of the channel was proposed.

In all the above research works, perfect channel state information (CSI) is assumed and coherent detection is employed at all receivers. In practice, this means channel estimation has to be done at the cooperative users and at the destination. This leads to a very complex system when many users are involved since channel estimation is needed for each pair of users, even if we neglect the possibility of asymmetric channels among the users.

In this paper, we propose a differential modulation scheme for two-user cooperative diversity systems which bypasses channel estimation. This modulation scheme can be considered as a nontrivial modification of differential phase-shift keying (DPSK) and differential space-time block codes (DSTBC) in [2]. Both users want to communicate to the same destination (e.g., base station). The information of one user stays in the in-phase axis (I axis), while that of the other user stays in the quadrature-phase axis ( $Q$ axis). This is to ensure that each user's information will be decodable at the destination. This paper considers the situation where both users have information to send and they share the same frequency band. Therefore, multiple access is done by time-division multiple access (TDMA). We focus on a half-duplex transmission, i.e., the mobiles cannot transmit and receive signals at the same time. In addition, the mobiles are able to perform differential decoding and re-encoding of the partner's message, as well as negative and conjugate operations before relaying the signals.

We consider the decode-and-forward and the selection relaying protocols [4] in which the relay mobiles perform differential decoding and re-encoding of the partner's message. In the 


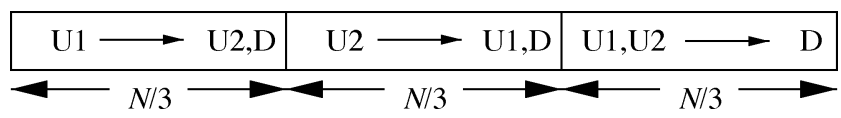

Fig. 1. TDMA scheme for two cooperative users, U1 is the first user, U2 is the second user, and $\mathrm{D}$ is the destination.

case of selection relaying, cyclic redundancy check (CRC) bits are used to detect whether the information has been received correctly. We derive the exact bit error probability (BEP) of the perfect interuser case which is a lower bound on the performance of the relaying protocols and the exact BEP of the selection relaying protocol in both asymmetric and symmetric interuser channels.

The paper is organized as follows. Section II describes the system model for a two-user cooperative diversity system. Section III proposes a differential modulation scheme for each cooperative user. Section IV presents the relaying protocols adapted from [4]. Section V presents performance analysis of the relaying protocols. Section VI presents the results and discussion. Concluding remarks are given in Section VII.

\section{SySTEM ModeL}

We consider a cellular system in which two cooperative users are transmitting their information to the same destination (e.g., the base station). A single antenna is accommodated at each user and at the destination. Both users share the same frequency band and the mobile of each user cannot transmit and receive a signal at the same time. The TDMA scheme and its associated transmissions and receptions are shown in Fig. 1. A total time frame is divided into three time frames. The first time frame belongs to the first user (U1) information. The second time frame belongs to the second user (U2) information. The third time frame is shared between both users and is used to relay each other's message to the destination (D). Notice that this partitioning of the time frame is different from those in [4] and [5] but similar to that in [10]. Compared with the space-time coded medium access control in [5], this partitioning is more bandwidth efficient since the relay transmissions share both time and frequency resources.

The interuser channels and the user-destination channels are independent of each other. All channels experience frequency flat fading and are quasi-static, i. e., they are fixed during a multiple-access time frame (three time frames) and change independently in the next frame. Suppose the multiple-access time frame occupies $N$ symbol intervals which accommodate $N_{f}=$ $N / 3-1$ information symbols from each user mobile.

With the above assumptions, we now consider a low-pass equivalent discrete-time model. In the first time frame, the first user mobile transmits its own message and the destination receiver and the second user mobile receive the signal. At the destination receiver, the received signal corresponding to the direct transmission $r_{1 d}[n]$ is given as

$$
r_{1 d}[n]=a_{1 d} s_{1}[n]+w_{d}[n]
$$

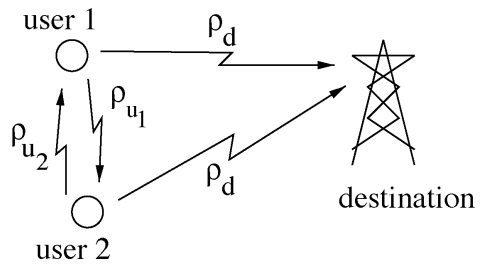

Fig. 2. SNR associated with each link.

where $s_{1}[n]$ is a symbol transmitted from the first user at the $n$th symbol interval, $n=0,1, \ldots,(N / 3)-1$. During this frame, at the second user mobile, the received signal $r_{12}[n]$ is written as

$$
r_{12}[n]=a_{12} s_{1}[n]+w_{2}[n] .
$$

In the second time frame, the roles of the first and the second user are switched. Therefore, at the destination receiver and at the first user mobile, the respective received signals $r_{2 d}[n]$ and $r_{21}[n]$ are

$$
r_{2 d}[n]=a_{2 d} s_{2}[n]+w_{d}[n], \quad r_{21}[n]=a_{21} s_{2}[n]+w_{1}[n]
$$

where $s_{2}[n]$ is a symbol transmitted from the second user at the $n$th symbol interval, $n=N / 3,(N / 3)+1, \ldots,(2 N / 3)-1$.

In the third time frame, both user mobiles act as relays and transmit the relay signals at the same time. The signal arriving at the destination is a linear combination of signals from both transmission paths, which can be written as

$$
r_{d}[n]=a_{1 d} s_{1 r}[n]+a_{2 d} s_{2 r}[n]+w_{d}[n]
$$

where $s_{1 r}[n], s_{2 r}[n]$ are relayed symbols transmitted from the first and the second user mobile, respectively, at the $n$th symbol interval $n=2 N / 3,(2 N / 3)+1, \ldots, N-1$.

In (1)-(4), the coefficients, $a_{i j}, i, j, \in\{1,2, d\}$, are associated with each transmission path from $i$ to $j$. They represent channel gains, which take into account fading and shadowing, and incorporate transmission signal energies. They are modeled as independent zero-mean complex Gaussian random variables. The subscripts " 1 " and " 2 " represent the first and the second user mobiles and the subscript " $d$ " represents the destination. Since the channels are fixed during the multiple-access time frame, the time variable $n$ is omitted. The $w_{i}[n], i \in\{1,2, d\}$ representing additive white noise and interference, are modeled as independent circularly symmetric zero-mean complex Gaussian random variables with variances $N_{i}$ and $i \in\{1,2, d\}$, respectively. The signal-to-noise ratios (SNRs) associated with each link shown in Fig. 2 are defined as $\rho_{d}=E\left[\left|a_{1 d}\right|^{2}\right] / N_{d}=E\left[\left|a_{2 d}\right|^{2}\right] / N_{d}$, $\rho_{u_{1}}=E\left[\left|a_{12}\right|^{2}\right] / N_{2}, \rho_{u 2}=E\left[\left|a_{21}\right|^{2}\right] / N_{1}$. The $\rho_{u_{1}}, \rho_{u_{2}}$ are referred to as interuser SNRs. For simplicity in the comparison with the no-cooperation case, both SNRs in the user-destination links are set to be equal to $\rho_{d}$.

\section{DIFFERENTIAL ENCODING AND DECODING}

To make this work, binary phase-shift keying (BPSK) information symbols are assumed for both users. As previously mentioned, the first user's data symbols lie on the I axis, while the second user's data symbols lie on the $\mathrm{Q}$ axis of the low-pass equivalent discrete-time model. The first and the second user's 
data symbols are represented, respectively, by $g_{1}[n]$ and $j \cdot g_{2}[n]$, where $g_{i}[n] \in\{-1,1\}, i=1,2$, and $j=\sqrt{-1}$. For the first user, the transmitted symbols in the first time frame are encoded by

$$
s_{1}[n]=g_{1}[n] s_{1}[n-1], \quad n=1,2, \ldots, \frac{N}{3}-1 .
$$

For the second user, the transmitted symbols in the second time frame are encoded by

$s_{2}[n]=j \cdot g_{2}[n] s_{2}[n-1], \quad n=\frac{N}{3}+1, \frac{N}{3}+2, \ldots, \frac{2 N}{3}-1$.

Note that while the transmission symbols of the first user are still in the BPSK constellation, the transmission symbols of the second user are expanded to the quadrature phase-shift keying (QPSK) constellation. The necessary initial symbols are assumed to be $s_{1}[0]=1$ and $s_{2}[N / 3]=1$. These a priori known initial symbols are needed to perform differential re-encoding at the relay mobiles.

Now, let us consider the transmission signals during the third time frame. In [4], a simple fixed amplify-and-forward protocol is shown to achieve spatial diversity, where the relay signal has been multiplied with a factor whose value depends on the channel gains and noise. Since we are not going to use CSI here, we choose to apply the decode-and-forward protocol in which differential decoding and re-encoding are performed at both users. At the first user mobile, differential decoding is performed by determining

$z_{2}[n]=r_{21}[n] r_{21}^{*}[n-1], \quad n=\frac{N}{3}+1, \frac{N}{3}+2, \ldots, \frac{2 N}{3}-1$

and the decoded data symbol, $\hat{g}_{2}[n]=1$, if $\operatorname{Im}\left(z_{2}[n] \geq 0\right)$ and $\hat{g}_{2}[n]=-1$, if $\operatorname{Im}\left(z_{2}[n]\right)<0$, where $\operatorname{Im}(\cdot)$ is the imaginary part and $*$ is a conjugate. The second user performs differential decoding by determining

$$
z_{1}[n]=r_{12}[n] r_{12}^{*}[n-1], \quad n=1,2, \ldots, \frac{N}{3}-1
$$

where the decoded data symbol, $\hat{g}_{1}[n]=1$, if $\operatorname{Re}\left(z_{1}[n]\right) \geq 0$ and $\hat{g}_{1}=-1$, if $\operatorname{Re}\left(z_{1}[n]\right)<0$. To perform differential re-encoding, the first user employs (6) with $\hat{g}_{2}[n]$ replacing $g_{2}[n]$. This results in a symbol $\hat{s}_{2}[n]$ which contains the second user's message. On the other hand, the second user employs (5) with $\hat{g}_{1}[n]$ replacing $g_{1}[n]$. This results in a symbol $\hat{s}_{1}[n]$ which contains the first user's message.

Now, the first user mobile transmits the relay signal as $s_{1 r}[n]=-\hat{s}_{2}^{*}[n-N / 3], n=2 N / 3,(2 N / 3)+1, \ldots, N-1$ and the second user mobile transmits the relay signal as $s_{2 r}[n]=\hat{s}_{1}^{*}[n-2 N / 3], n=2 N / 3,(2 N / 3)+1, \ldots, N-1$ simultaneously. This structure is similar to the Alamouti's scheme in which the direct transmission symbols correspond to the elements in the first row (symbol interval), while the relayed symbols correspond to the elements in the second row of the Alamouti's matrix [1]. The received signal in (4) can be rewritten as

$$
\begin{array}{r}
r_{d}[n]=-a_{1 d} \hat{s}_{2}^{*}\left[n-\frac{N}{3}\right]+a_{2 d} \hat{s}_{1}^{*}\left[n-\frac{2 N}{3}\right]+w_{d}[n], \\
n=\frac{2 N}{3}, \frac{2 N}{3}+1, \ldots, N-1 .
\end{array}
$$

After this signal arrives at the destination, differential decoding is performed as follows. First, the receiver combines the signals from the direct transmissions from both users as

$$
\begin{aligned}
r_{c}[n]= & r_{1 d}\left[n-\frac{2 N}{3}\right]+r_{2 d}\left[n-\frac{N}{3}\right] \\
= & a_{1 d} s_{1}\left[n-\frac{2 N}{3}\right]+a_{2 d} s_{2}\left[n-\frac{N}{3}\right] \\
& +w_{d}\left[n-\frac{2 N}{3}\right]+w_{d}\left[n-\frac{N}{3}\right]
\end{aligned}
$$

where $n=2 N / 3,(2 N / 3)+1, \ldots, N-1$. The received signals in (9) and (10) are almost similar to those of the Alamouti's scheme. The only differences are another additive noise term in (10), the possibility of symbol errors in (9), and the delay of user symbols inherent in the cooperative system. Next, the receiver computes

$$
z_{d}[n]=r_{c}[n] r_{c}^{*}[n-1]+r_{d}^{*}[n] r_{d}[n-1]
$$

where $n=(2 N / 3)+1,(2 N / 3)+2, \ldots, N-1$. Substituting (9) and (10) into (11) and after some manipulation, we can readily show that

$$
\begin{aligned}
z_{d}[n]= & \left|a_{1 d}\right|^{2}\left(s_{1}\left[n-\frac{2 N}{3}\right] s_{1}^{*}\left[n-\frac{2 N}{3}-1\right]\right. \\
& \left.+\hat{s}_{2}\left[n-\frac{N}{3}\right] \hat{s}_{2}^{*}\left[n-\frac{N}{3}-1\right]\right) \\
& +\left|a_{2 d}\right|^{2}\left(\hat{s}_{1}\left[n-\frac{2 N}{3}\right] \hat{s}_{1}^{*}\left[n-\frac{2 N}{3}-1\right]\right. \\
& \left.\quad+s_{2}\left[n-\frac{N}{3}\right] \hat{s}_{2}^{*}\left[n-\frac{N}{3}-1\right]\right) \\
& +I+\text { noise } \\
= & \left|a_{1 d}\right|^{2}\left(g_{1}\left[n-\frac{2 N}{3}\right]+j \cdot \hat{g}_{2}\left[n-\frac{N}{3}\right]\right) \\
& +\left|a_{2 d}\right|^{2}\left(\hat{g}_{1}\left[n-\frac{2 N}{3}\right]+j \cdot g_{2}\left[n-\frac{N}{3}\right]\right) \\
& +I+\text { noise }
\end{aligned}
$$

where $I=a_{1 d} a_{2 d}^{*}\left(s_{1}[n-2 N / 3] s_{2}^{*}[n-N / 3-1]-\hat{s}_{1}[n-\right.$ $\left.2 N / 3] \hat{s}_{2}^{*}[n-N / 3-1]\right)+a_{1 d}^{*} a_{2 d}\left(s_{1}^{*}[n-2 N / 3-1] s_{2}[n-\right.$ $\left.N / 3]-\hat{s}_{1}^{*}[n-2 N / 3-1] \hat{s}_{2}[n-N / 3]\right)$. Note that $I=0$ only when both relay mobiles transmit correct re-encoded symbols. Since the data symbols $g_{1}[n-2 N / 3]$ and $j \cdot g_{2}[n-N / 3]$ are in the I axis and Q axis, respectively, we can decode them separately by considering the real and imaginary parts of (12). Therefore, the following decision rules are obtained:

$$
\begin{aligned}
\tilde{g}_{1}\left[n-\frac{2 N}{3}\right] & =\left\{\begin{array}{ll}
1, & \text { if } \operatorname{Re}\left(z_{d}[n]\right)>0 \\
-1, & \text { else }
\end{array}\right. \text { and } \\
\tilde{g}_{2}\left[n-\frac{N}{3}\right] & = \begin{cases}1, & \text { if } \operatorname{Im}\left(z_{d}[n]\right)>0 \\
-1, & \text { else }\end{cases}
\end{aligned}
$$

where $n=(2 N / 3)+1,(2 N / 3)+2, \ldots, N-1$.

Note that the differential decoding in (12) is almost similar to the differential detection for DSTBC in [2]. The difference is that more noise terms exist in (12) and neither $g_{1}[n-2 N / 3]+j$. $\hat{g}_{2}[n-N / 3]$ nor $\hat{g}_{1}[n-2 N / 3]+j \cdot g_{2}[n-N / 3]$ represents a single symbol (as in the case of DSTBC). Instead, each represents two symbols, $\left\{g_{1}[n-2 N / 3], j \cdot g_{2}[n-N / 3]\right\}$ transmitted from two users and from different axes, as well as the relay symbols which 
have been re-encoded. If the relay symbols are re-encoded at the mobiles without error, i.e., $\hat{g}_{1}[n-2 N / 3]=g_{1}[n-2 N / 3]$, $\hat{g}_{2}[n-N / 3]=g_{2}[n-N / 3]$, at the destination, it seems that each data symbol experiences a second-order diversity (from $\left|a_{1 d}\right|^{2}$ and $\left|a_{2 d}\right|^{2}$ ). Therefore, at high interuser SNRs, we expect performance improvement due to this spatial diversity from the cooperative user channels. Note that for this scheme to work, the symbol $g_{1}[n-2 N / 3]+j \cdot g_{2}[n-N / 3]$ must be unique and has the same Euclidean norm. This limits our use to the BPSK information. One might ask why DSTBC can be applied with higher order M-PSK symbols. The reason is that DSTBC has another decoding metric ([2, eq. (23)]) to make M-PSK decoding possible. Unfortunately, this metric does not have obvious physical meaning when applying this to the information symbols of the cooperative users and, therefore, it cannot be used here.

\section{RELAYING PROTOCOLS}

In the cooperative diversity system, the relaying protocol is a crucial component to achieve diversity. Each protocol has its own tradeoffs among performance, complexity and data transmission rate. The relaying protocols in this paper are adapted from [4].

\section{A. Fixed Decode-and-Forward Relaying (DF)}

With this protocol, the relay mobile fully decodes or symbol-by-symbol decodes the received codeword before transmitting the relay signal. In [4], "decode" refers to coherent detection and "forward" refers to relaying the decoded symbols. In this paper, "decode" means differential decoding and "forward" means relaying the re-encoded symbols as described in the previous section.

\section{B. Selection Relaying (SR)}

When the channels between cooperative users are not good, several decoded and re-encoded symbol errors occur at the relay mobiles. With the DF, it will turn out that several relay symbols in error will be transmitted. These relay symbols in error result in performance degradation even when the direct transmission channels are good. Therefore, it turns out that it is better not to transmit anything from the relay mobile when the interuser channels are not good. In [4], SR was proposed in which not all relay mobiles are allowed to transmit the relay signals. The permission to transmit the relay signals or not depends on the amplitudes of the fading gains between the source mobile and the relay mobile. If these values fall below a certain threshold, the relay mobile is not allowed to transmit the relay signal but instead transmits its own information signal. In this paper, since we are not going to use the CSI, we assume that CRC bits have been added before differential encoding at the source mobile. Then, at the relay mobile, the receiver performs a CRC check after differential decoding to see if the whole frame has been received correctly. If the frame is received correctly, the relay mobile is allowed to perform differential re-encoding and forward the relay signal, otherwise, it does nothing. The relay mobile is not allowed to transmit its own message at this time frame since doing that will destroy the Alamouti's transmission structure and differential decoding operation. The CRC check technique is also used in [7] with the possibility of continuing its own transmission of the relay mobile. In this paper, we assume that 16-bit CRC is applied and its error detection is perfect.

When one of the mobiles stops its relay transmission, the destination will adapt the decoding scheme in order to avoid noise from its direct transmission. For example, suppose only the second user mobile transmits the relay signal whose information belongs to the first user mobile. For the destination to decode the first user's message, the direct transmission signal from the first user in (1) instead of the combined signal in (10) will be used to compute the decision variable in (11), while conventional DPSK differential decoding is used for the second user. In this manner, diversity can still be achieved for the first user mobile without being interfered by the second user direct transmission.

Note that both protocols require the destination to know whose message the user is transmitting. We have to resort this problem to the higher layer protocol. The message may be sent by a control channel/protocol information. Note that this message has to be sent only once to the destination since frames are synchronized.

\section{Performance Analysis}

The performance measure in this paper is BEP. Since differential decoding in this paper has an intimate relationship to DSTBC decoding, the analysis for DSTBC can be readily applied in the cooperative system. In this paper, we follow the approach in [11], where the BEPs of DSTBC with BPSK and QPSK were derived. The analysis here includes both the symmetric and asymmetric interuser cases.

\section{A. BEP in the Perfect Interuser Case}

When the cooperative users have perfect information about each other's transmission sequence, there will be no misleading relay information transmitted. This ideal case represents the limiting performance of cooperative diversity with any kind of relaying protocols. Therefore, the performance of the perfect interuser information case will be a lower bound on the performance of the DF and SR protocols in this paper. This bound will also be used in the analysis of the SR protocol to follow. In the perfect interuser case, the BEP does not depend on the interuser channel. It rather depends on how the destination decodes the signals. For the SR protocol, it might happen that one of the mobiles stops its relay transmission (one user does not have perfect information of the other). Therefore, the computation of the BEP is divided into two cases.

1) Full Relaying: Full relaying occurs when both user mobiles transmit their relay signals. For a single receive antenna at the destination, the BEP is given by (see Appendix I)

$$
P_{b, \text { full relaying }}=\frac{1}{2}\left[1-\sqrt{\frac{\rho_{d}}{\rho_{d}+6}}\left(\frac{\rho_{d}+9}{\rho_{d}+6}\right)\right] \text {. }
$$

2) Single Relaying: When one of the user mobiles stops its relay transmission, the destination receives only the relay signal 
from the other user. For a single receive antenna at the destination, the BEP is given by (see Appendix I)

$$
P_{b, \text { single relaying }}=\frac{1}{2}\left[1-\sqrt{\frac{\rho_{d}}{\rho_{d}+2}}\left(\frac{\rho_{d}+3}{\rho_{d}+2}\right)\right] .
$$

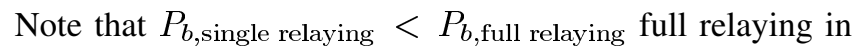
general. This makes sense since the number of noise terms in (12) is smaller in the single relaying case (compare (19) and (24)), i.e., performance is gained at the user with single relaying without being interfered by the other user.

\section{B. BEP of SR Protocol}

In the SR protocol, a user mobile transmits the relay signal when the whole frame has been received correctly. This implies that no symbols in error will be transmitted in the relay signal, and we can apply the BEP derived in the perfect interuser case. This also means that the probability of a user mobile transmitting the relay signal is $\left(1-P_{F, \text { DPSK, } u_{i}}\right)$, where $P_{F, \text { DPSK }, u_{i}}$ is the frame error probability of DPSK belonging to the $i$ th user mobile. The frame error probability of DPSK is given by (see Appendix II)

$$
P_{F, \text { DPSK }, u_{i}}=1-\left(\frac{1}{2}\right)^{N_{f}}\left[1+\sum_{k=1}^{N_{f}} \prod_{l=1}^{k} \frac{N_{f}+1-l}{l+\frac{l}{\rho_{u_{i}}}}\right]
$$

Without loss of generality, let us consider the BEP of the first user. The following four cases can occur.

Case 1) No users transmit a relay signal: The probability of this case is $P_{F, \text { DPSK }, u_{1}} \cdot P_{F, \text { DPSK }, u_{2}}$. Bit errors occurring at the destination are from DPSK decoding of the direct transmission signal belonging to the first user.

Case 2) Only the second user transmits a relay signal: The probability of this case is $\left(1-P_{F, \mathrm{DPSK}, u_{1}}\right)$. $P_{F, \text { DPSK }, u_{2}}$. Bit errors occurring at the destination are from differential decoding with single relaying.

Case 3) Only the first user transmits a relay signal: The probability of this case is $P_{F, \text { DPSK, } u_{1}} \cdot(1-$ $\left.P_{F, \text { DPSK }, u_{2}}\right)$. Bit errors occurring at the destination are from DPSK decoding similar to the first case.

Case 4) Both users transmit a relay signal: The probability of this case is $\left(1-P_{F, \text { DPSK }, u_{1}}\right) \cdot\left(1-P_{F, \text { DPSK, } u_{2}}\right)$. Bit errors occurring at the destination are from differential decoding with full relaying.

The BEP of the SR protocol is found by averaging BEPs of the above four cases. This can be written as

$$
\begin{aligned}
P_{b, \mathrm{SR}}= & P_{F, \mathrm{DPSK}, u_{1}} \cdot P_{F, \mathrm{DPSK}, u_{2}} \cdot P_{b, \mathrm{DPSK}} \\
& +\left(1-P_{F, \mathrm{DPSK}, u_{1}}\right) \cdot P_{F, \mathrm{DPSK}, u_{2}} \cdot P_{b, \text { single relaying }} \\
& +P_{F, \mathrm{DPSK}, u_{1}} \cdot\left(1-P_{F, \mathrm{DPSK}, u_{2}}\right) \cdot P_{b, \mathrm{DPSK}} \\
& +\left(1-P_{F, \mathrm{DPSK}, u_{1}}\right) \cdot\left(1-P_{F, \mathrm{DPSK}, u_{2}}\right) \\
& \cdot P_{b, \text { full relaying }}
\end{aligned}
$$

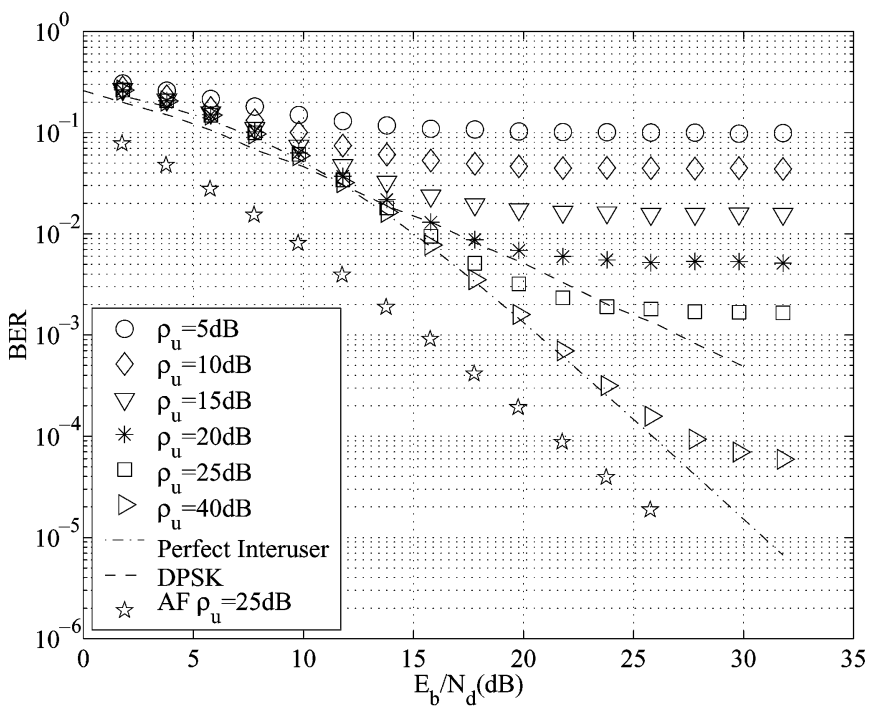

Fig. 3. BPSK differential modulation with fixed decode-and-forward relaying protocol at various interuser SNRs under symmetric interuser channels. Simulation and analytical results are shown by markers and lines, respectively.

where $P_{b, \mathrm{DPSK}}=1 /\left(1+2 \rho_{d}\right)$ is a conventional BEP of DPSK under Rayleigh-fading channels [12]. When the $\rho_{u 1}=\rho_{u 2}=$ $\rho_{u},(16)$ reduces to

$$
\begin{gathered}
P_{b, \mathrm{SR}}=P_{F, \mathrm{DPSK}, u} \cdot P_{b, \mathrm{DPSK}}+\left(1-P_{F, \mathrm{DPSK}, u}\right) \cdot P_{F, \mathrm{DPSK}, u} \\
\cdot P_{b, \text { single relaying }}+\left(1-P_{F, \mathrm{DPSK}, u}\right)^{2} \cdot P_{b, \text { full relaying }}
\end{gathered}
$$

in which the first user and the second user are not distinguished.

\section{RESULTS AND DisCUSSION}

Noncooperative transmission of DPSK is chosen as a baseline performance which does not have diversity gain. Included in all plots are the performance of DPSK, the performance with perfect interuser information and full relaying (for DF or SR protocols), and the performance of amplify-and-forward relaying (AF) with coherent detection [4], [13]. In order to compare different protocols with a different rate loss due to a reference symbol and CRC bits, let us define the pure information bit SNR as

$$
\frac{E_{b}}{N_{d}}=\frac{\rho_{d}}{R} \cdot \frac{N_{f}+1}{N_{f}+1-\gamma}
$$

where $R=1, \gamma=0$ for DPSK, $R=2 / 3, \gamma=0$ for the AF protocol, $R=2 / 3, \gamma=1$ for the DF protocol and $R=$ $2 / 3, \gamma=1+$ no. of CRC bits for the SR protocol. The frame length $\left(N_{f}+1\right)$ is 131 symbol intervals which accommodate 130 information symbols. In the figures, simulation results are presented with markers, while analytical results are presented with lines.

\section{A. Symmetric Interuser Channels}

In this case, since all the channels seen by each user are the same, both users experience the same performance. As shown in Fig. 3, the DF protocol achieves a performance gain over DPSK when $\rho_{u}>20 \mathrm{~dB}$ in the medium range of $E_{b} / N_{d}$. At $E_{b} / N_{d}$ relatively higher than $\rho_{u}$, the protocol experiences an 


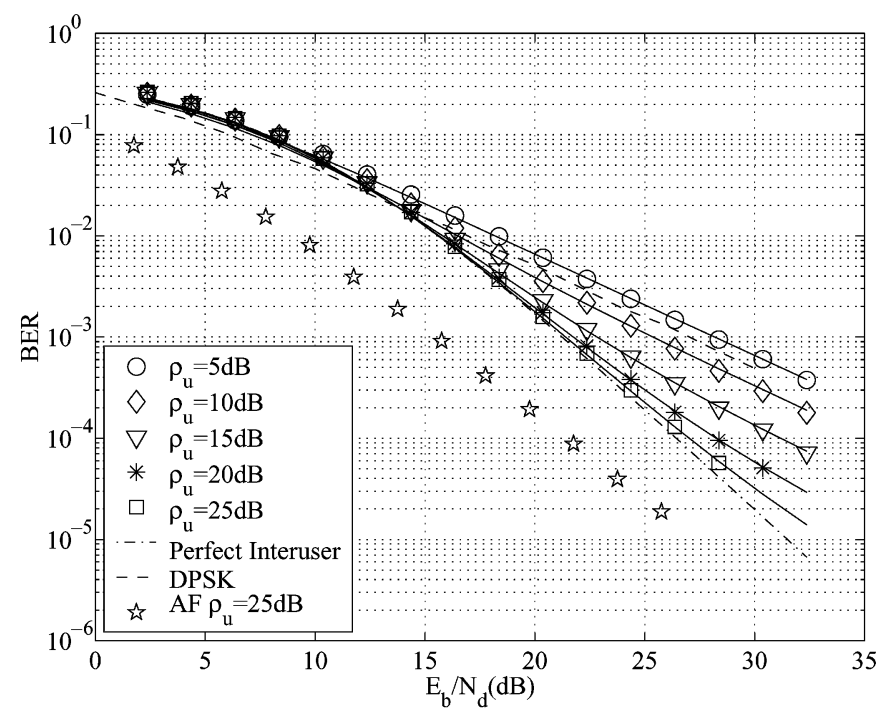

Fig. 4. BPSK differential modulation with selection relaying protocol at various interuser SNRs under symmetric interuser channels. Simulation and analytical results are shown by markers and lines, respectively.

error floor and cannot outperform DPSK. This error floor comes from the re-encoded symbols in error occurring at the relay mobile. These re-encoded symbols in error cause misleading information which affects the decoding in (12). When $\rho_{u}$ is very high, e.g., $\rho_{u}=40 \mathrm{~dB}$, the protocol outperforms DPSK over the whole $E_{b} / N_{d}$ range of interest. In the perfect interuser case, no error floor exists and it indeed achieves second-order diversity as seen from the slope of the curve. The analytical BEP matches exactly with the simulation results (not shown) in the perfect interuser case. The AF protocol with coherent detection has about 4-dB performance gain compared with even the perfect interuser case at BEP of $10^{-4}$.

The performance of the SR protocol is shown in Fig. 4. At $\rho_{u}=5 \mathrm{~dB}$, the protocol is about 1-dB inferior to DPSK mainly due to the rate loss caused by CRC bits. At $\rho_{u}=10 \mathrm{~dB}$, the protocol achieves about 2-dB gain over DPSK at medium to high $E_{b} / N_{d}$. At $\rho_{u}=25 \mathrm{~dB}$, the protocol has only less than a 1-dB gap compared with the perfect interuser case at $\mathrm{BEP}=10^{-4}$. No error floor is found in the SR protocol since no symbols in error are allowed to be transmitted in the relay signals. The analytical results show excellent matches with the simulation results. Note that the gap between the AF protocol and the perfect interuser case with the SR protocol is a little greater than that with the DF protocol since CRC bits in the SR protocol introduces a small rate loss.

\section{B. Asymmetric Interuser Channels}

When the interuser channels are asymmetric, each user experiences a different performance. This might occur when one user experiences local interference, while the other does not. For example, one user might be surrounded by local interfering devices or mobiles which do not affect the other user since the distance is too far. The asymmetric interuser case can also occur when power control is used and due to different distances from the base station, the users' transmit powers could be different. We fix $\rho_{u_{1}}=25 \mathrm{~dB}$ and evaluate the performance of both users when $\rho_{u_{2}}=15,20 \mathrm{~dB}$. The first user's performance is shown

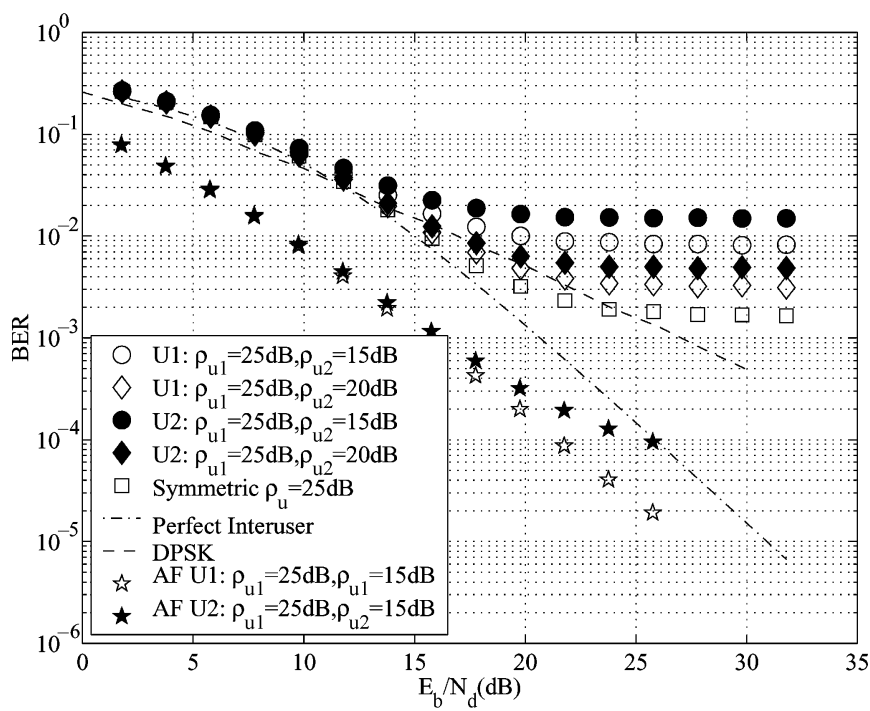

Fig. 5. BPSK differential modulation with fixed decode-and-forward relaying protocol under asymmetric interuser channels. Simulation and analytical results are shown by markers and lines, respectively.

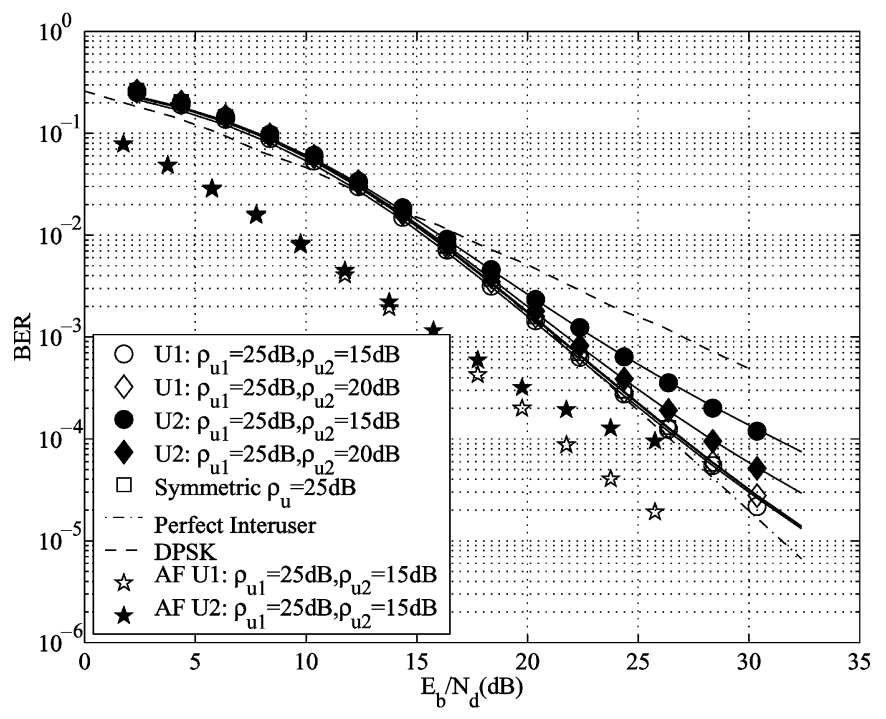

Fig. 6. BPSK differential modulation with selection relaying protocol under asymmetric interuser channels. Simulation and analytical results are shown by markers and lines, respectively.

by empty markers and the second user's performance is shown by filled markers. With the DF protocol, as shown in Fig. 5, the first user experiences a performance degradation from the asymmetric interuser channels. The error floor of the first user is higher when $\rho_{u_{2}}$ is lower. This is because symbols in error belonging to the second user presented in the relay signal affect the decoding of the first user as well [see (12)]. From the second user's point of view, a higher $\rho_{u_{1}}$ does not provide any help to the second user's performance. For example, the performance of the second user mobile at $\rho_{u_{2}}=20 \mathrm{~dB}$ stays the same whether when $\rho_{u_{1}}$ is at $25 \mathrm{~dB}$ or at $20 \mathrm{~dB}$. (Compare Figs. 3 and 5.) The AF protocol largely outperforms the DF protocol in this asymmetric channel case.

For the SR protocol, as shown in Fig. 6, the performance of the first user is not deteriorated when $\rho_{u 2}<\rho_{u 1}$. In fact, a small improvement of the first user is observed when $\rho_{u_{2}}$ is lower. 
Since when this situation happens, the first user mobile transmits the relay signal less often and the BEP at the destination falls more frequently into the single relaying case whose BEP is lower than that of the full relaying case. The above discussion implies that the SR protocol is robust to asymmetric interuser channels. Similar to the DF protocol, from the second user's point of view, $\rho_{u 1}>\rho_{u 2}$ does not provide any help to the second user's performance. Compared with the AF protocol, both users have about 5-dB gain over the SR protocol at BEP of $10^{-4}$.

\section{CONCLUDING REMARKS}

A novel differential modulation scheme is proposed in a twouser cooperative diversity system which does not require CSI either at the user mobiles or at the destination. This scheme achieves second-order diversity efficiently with the selection relaying protocols. With the DF, this scheme still significantly outperforms DPSK when interuser SNR is high and the interuser channels are symmetric. We have shown that the selection relaying protocol is robust to the asymmetric interuser channels by both simulation and analytical results. Although AF seems to outperform the proposed approach, the first requires CSI, whereas the latter does not. Extension to more than two cooperative users would be a challenging task.

Note that the effect of phase noise to the proposed scheme remains to be investigated. Nevertheless, we expect that if the phase noise associated with each user-destination link is symmetric and small, our approach should tolerate a certain amount of phase noise. However, if the phase noise associated with each user is different (asymmetric), the proposed scheme might have considerable amount of performance degradation. This is in contrast to DSTBC in which the antennas are co-located and they can share the same oscillator.

During the revision of this paper, we found that [14] addresses noncoherent approach for cooperative diversity system as well. However, their approach is based on BFSK and only one user source exists.

\section{APPENDIX I}

\section{BEP OF DifFERENTIAL MODULATION IN THE PERFECT INTERUSER CASE}

Without loss of generality, the data symbols are assumed to be $g_{1}[n-2 N / 3]=1, g_{2}[n-N / 3]=1$. The decision variable is computed during the time interval $n=(2 N / 3)+1,(2 N / 3)+$ $2, \ldots, N-1$. In the following derivations, we will neglect all second-order noise terms. Due to the symmetry of the constellation, the BEP in other cases can be found to be the same.

\section{A. Full Relaying}

In this case, the decision variable $z_{d}[n]$ from (12) at the destination can be written as

$$
\begin{aligned}
z_{d}[n]= & \left(\left|a_{1 d}\right|^{2}+\left|a_{2 d}\right|^{2}\right)\left(g_{1}\left[n-\frac{2 N}{3}\right]+j \cdot g_{2}\left[n-\frac{N}{3}\right]\right) \\
& +a_{1 d}\left(s_{1}\left[n-\frac{2 N}{3}\right] w_{d}^{*}\left[n-\frac{2 N}{3}-1\right]+s_{1}\left[n-\frac{2 N}{3}\right]\right. \\
& \times w_{d}^{*}\left[n-\frac{N}{3}-1\right]-s_{2}^{*}\left[n-\frac{N}{3}-1\right] w_{d}^{*}[n]
\end{aligned}
$$

$$
\begin{aligned}
+ & s_{1}^{*}\left[n-\frac{2 N}{3}-1\right] v_{1} w_{d}\left[n-\frac{2 N}{3}\right] \\
& +s_{1}^{*}\left[n-\frac{2 N}{3}-1\right] v_{1} w_{d}\left[n-\frac{N}{3}\right] \\
& \left.-s_{2}\left[n-\frac{N}{3}\right] v_{1} w_{d}[n-1]\right) \\
+a_{2 d}( & s_{2}\left[n-\frac{n}{3}\right] w_{d}^{*}\left[n-\frac{2 N}{3}-1\right]+s_{2}\left[n-\frac{n}{3}\right] \\
& \times w_{d}^{*}\left[n-\frac{N}{3}-1\right]+s_{1}^{*}\left[n-\frac{2 N}{3}-1\right] w_{d}^{*}[n] \\
& +s_{2}^{*}\left[n-\frac{N}{3}-1\right] v_{2} w_{d}\left[n-\frac{2 N}{3}\right] \\
& +s_{2}^{*}\left[n-\frac{N}{3}-1\right] v_{2} w_{d}\left[n-\frac{N}{3}\right] \\
& \left.+s_{1}\left[n-\frac{2 N}{3}\right] v_{2} w_{d}[n-1]\right)
\end{aligned}
$$

where $v_{1}=a_{1 d}^{*} / a_{1 d}, v_{2}=a_{2 d}^{*} / a_{2 d}$. Note that the multiplication of $v_{i}$ does not affect the probability density function (pdf) of noise. The symbols from both users are represented by $\lambda=g_{1}[n-2 N / 3]+j \cdot g_{2}[n-N / 3]$, which can be viewed as a single symbol with amplitude $\sqrt{2}$ in a two-dimensional constellation shown in Fig. 7. Define the following variables:

$$
\begin{aligned}
X_{1}= & a_{1 d} \\
X_{2}= & a_{2 d} \\
Y_{1}= & a_{1 d} \lambda^{*}+s_{1}^{*}\left[n-\frac{2 N}{3}\right] w_{d}\left[n-\frac{2 N}{3}-1\right] \\
& +s_{1}^{*}\left[n-\frac{2 N}{3}\right] w_{d}\left[n-\frac{N}{3}-1\right]-s_{2}\left[n-\frac{N}{3}-1\right] w_{d}[n] \\
& +s_{1}\left[n-\frac{2 N}{3}-1\right] v_{1}^{*} w_{d}^{*}\left[n-\frac{2 N}{3}\right] \\
& +s_{1}\left[n-\frac{2 N}{3}-1\right] v_{1}^{*} w_{d}^{*}\left[n-\frac{N}{3}\right] \\
& -s_{2}^{*}\left[n-\frac{N}{3}\right] v_{1}^{*} w_{d}^{*}[n-1] \\
Y_{2}= & a_{2 d} \lambda^{*}+s_{2}^{*}\left[n-\frac{N}{3}\right] w_{d}\left[n-\frac{2 N}{3}-1\right] \\
& +s_{2}^{*}\left[n-\frac{N}{3}\right] w_{d}\left[n-\frac{N}{3}-1\right]+s_{1}\left[n-\frac{2 N}{3}-1\right] w_{d}[n] \\
& +s_{2}\left[n-\frac{N}{3}-1\right] v_{2}^{*} w_{d}^{*}\left[n-\frac{2 N}{3}\right] \\
& +s_{2}\left[n-\frac{N}{3}-1\right] v_{2}^{*} w_{d}^{*}\left[n-\frac{N}{3}\right] \\
& +s_{1}^{*}\left[n-\frac{2 N}{3}\right] v_{2}^{*} w_{d}^{*}[n-1] .
\end{aligned}
$$

The decision variable is written as $z_{d}[n]=X_{1} Y_{1}^{*}+X_{2} Y_{2}^{*}$. We also have $m_{x x}=E\left[X_{1} X_{1}^{*}\right]=E\left[X_{2} X_{2}^{*}\right]=1$, $m_{y y}=E\left[Y_{1} Y_{1}^{*}\right]=E\left[Y_{2} Y_{2}^{*}\right]=2+6 / \rho_{d}, m_{x y}=$ $E\left[X_{1} Y_{1}^{*}\right]=E\left[X_{2} Y_{2}^{*}\right]=\sqrt{2}, E\left[X_{1} X_{2}^{*}\right]=E\left[Y_{1} Y_{2}^{*}\right]=$ $E\left[X_{1} Y_{2}^{*}\right]=E\left[X_{2} Y_{1}^{*}\right]=0$. Therefore, $\left(X_{1}, Y_{1}\right)$ and $\left(X_{2}, Y_{2}\right)$ are zero-mean correlated complex Gaussian random variables with each pair independent and identically distributed from the 


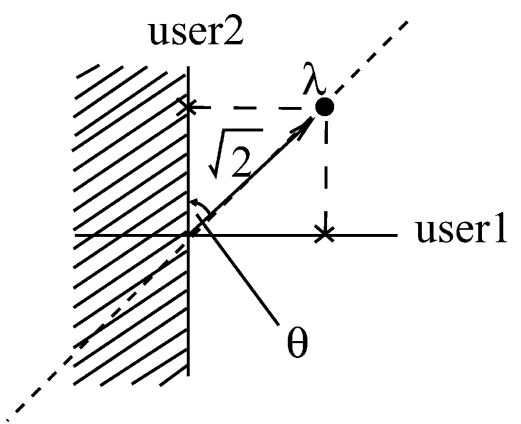

Fig. 7. Inegratino region of the BEP in the perfect interuser information case with full relaying.

other. Now, the problem at hand closely matches with those in [11] and [12, App. C].

The symbol $\lambda$ can be considered as that it lies on the $\pi / 4$-rotated constellation with respect to the real axis, as shown in Fig. 7. Hence, the BEP is the probability of the phase of $z_{d}[n]$, defined as $\theta$, lying between $\pi / 4$ and $5 \pi / 4$. Since the pdf of $\theta$, $p(\theta)$, is an even function [12], the BEP can be expressed as

$$
P_{b, \text { full relaying }}=P\left(\frac{\pi}{4} \leq \theta \leq \frac{5 \pi}{4}\right)=\int_{\frac{\pi}{4}}^{\frac{3 \pi}{4}} p(\theta) d \theta+2 \int_{\frac{3 \pi}{4}}^{\pi} p(\theta) d \theta .
$$

This integral has the same form as the BEP of QPSK under Rayleigh-fading channels [11], [12], which has been evaluated as

$$
P_{b, \text { full relaying }}=\frac{1}{2}\left[1-\frac{\mu}{\sqrt{2-\mu^{2}}} \sum_{k=0}^{2 N_{r}-1}\left(\begin{array}{c}
2 k \\
k
\end{array}\right)\left(\frac{1-\mu^{2}}{4-2 \mu^{2}}\right)^{k}\right] .
$$

Here, $N_{r}$ is the number of receive antennas, $\mu=$ $m_{x y} / \sqrt{m_{x x} m_{y y}}=1 / \sqrt{1+3 / \rho_{p}}$. For one receive antenna, substituting $\mu$ into (22), we obtain

$$
P_{b, \text { full relaying }}=\frac{1}{2}\left[1-\sqrt{\frac{\rho_{d}}{\rho_{d}+6}}\left(\frac{\rho_{d}+9}{\rho_{d}+6}\right)\right] .
$$

\section{B. Single Relaying}

Suppose only the second user transmits a relay signal whose information belongs to the first user. Then, the decision variable $z_{d}[n]$ can be written as

$$
\begin{aligned}
z_{d}[n]= & \left(\left|a_{1 d}\right|^{2}+\left|a_{2 d}\right|^{2}\right)\left(g_{1}\left[n-\frac{2 N}{3}\right]\right) \\
+ & a_{1 d}\left(s_{1}\left[n-\frac{2 N}{3}\right] w_{d}^{*}\left[n-\frac{2 N}{3}-1\right]\right. \\
& \left.\quad+s_{1}^{*}\left[n-\frac{2 N}{3}-1\right] v_{1} w_{d}\left[n-\frac{2 n}{3}\right]\right) \\
+ & a_{2 d}\left(s_{1}^{*}\left[n-\frac{2 N}{3}-1\right] w_{d}^{*}[n]\right. \\
& \left.+s_{1}\left[n-\frac{2 N}{3}\right] v_{2} w_{d}[n-1]\right) .
\end{aligned}
$$

Similar to the full relaying case, let us define

$$
\begin{aligned}
X_{1}= & a_{1 d} \\
X_{2}= & a_{2 d} \\
Y_{1}= & a_{1 d} g_{1}^{*}\left[n-\frac{2 N}{3}\right]+s_{1}^{*}\left[n-\frac{2 N}{3}\right] w_{d}\left[n-\frac{2 N}{3}-1\right] \\
& +s_{1}\left[n-\frac{2 N}{3}-1\right] v_{1}^{*} w_{d}^{*}\left[n-\frac{2 N}{3}\right] \\
Y_{2}= & a_{2 d} g_{1}^{*}\left[n-\frac{2 N}{3}\right]+s_{1}\left[n-\frac{2 N}{3}-1\right] w_{d}[n] \\
& +s_{1}^{*}\left[n-\frac{2 N}{3}\right] v_{2}^{*} w_{d}^{*}[n-1] .
\end{aligned}
$$

The decision variable is written as $z_{d}[n]=X_{1} Y_{1}^{*}+X_{2} Y_{2}^{*}$. In addition, we also have $m_{x x}=E\left[X_{1} X_{1}^{*}\right]=E\left[X_{2} X_{2}^{*}\right]=1$, $m_{y y}=E\left[Y_{1} Y_{1}^{*}\right]=E\left[Y_{2} Y_{2}^{*}\right]=1+2 / \rho_{d}, m_{x y}=E\left[X_{1} Y_{1}^{*}\right]=$ $E\left[X_{2} Y_{2}^{*}\right]=1, E=\left[X_{1} X_{2}^{*}\right]=E\left[Y_{1} Y_{2}^{*}\right]=E\left[X_{1} Y_{2}^{*}\right]=$ $E\left[X_{2} Y_{1}^{*}\right]=0$. Therefore, $\left(X_{1}, Y_{1}\right)$ and $\left(X_{2}, Y_{2}\right)$ are zero-mean correlated complex Gaussian random variables with each pair independent and identically distributed from the other.

Hence, the BEP is the probability of $\theta$ lying between $\pi / 2$ and $3 \pi / 2$ which is expressed as

$$
P_{b, \text { single relaying }}=P\left(\frac{\pi}{2} \leq \theta \leq \frac{3 \pi}{2}\right)=2 \int_{\frac{\pi}{2}}^{\pi} p(\theta) d \theta .
$$

This integral has a similar form as BEP for BPSK under Rayleigh-fading channels which has been evaluated as [11], [12]

$$
P_{b, \text { single relaying }}=\frac{1}{2}\left[1-\mu \sum_{k=0}^{2 N_{r}-1}\left(\begin{array}{c}
2 k \\
k
\end{array}\right)\left(\frac{1-\mu^{2}}{4}\right)^{k}\right] .
$$

Here, $\mu=m_{x y} / \sqrt{m_{x x} m_{y y}}=1 / \sqrt{1+2 / \rho_{d}}$. For one receive antenna, substituting $\mu$ into (27), we obtain

$$
P_{b, \text { single relaying }}=\frac{1}{2}\left[1-\sqrt{\frac{\rho_{d}}{\rho_{d}+2}}\left(\frac{\rho_{d}+3}{\rho_{d}+2}\right)\right] .
$$

\section{APPENDIX II}

FrAME ERROR PROBABILITY OF DPSK UNDER QUASI-STATIC RAYLEIGH-FADING CHANNELS

For given fixed channel gains within a frame (and therefore, fixed SNR), the BEP of DPSK as a function of SNR is given by [12]

$$
P_{b, \mathrm{DPSK}}(\rho)=\frac{1}{2} e^{-\rho}
$$

where $\rho$ is a given SNR. Since the occurrence of each bit error is independent for a given SNR, the frame error probability is given by

$$
P_{F, \operatorname{DPSK}}(\rho)=1-\left(1-\frac{1}{2} e^{-\rho}\right)^{N_{f}}
$$

where $N_{f}$ is the information frame length. The frame error probability of DPSK under quasi-static Rayleigh-fading channels can be found by averaging (30) over the pdf of SNR. The pdf 
of instantaneous SNR under Rayleigh-fading channel is given by $p(\rho)=(1 / \bar{\rho}) e^{-\rho / \bar{\rho}}, \rho \geq 0$ [12]. Hence, the required frame error probability is

$$
P_{F, \text { DPSK }}=\int_{0}^{\infty}\left[1-\left(1-\frac{1}{2} e^{-\rho}\right)^{N_{f}}\right]\left(\frac{1}{\bar{\rho}} e^{-\frac{\rho}{\bar{\rho}}}\right) d \rho=1-\mathcal{I}
$$

where $\mathcal{I}$ is defined as

$$
\mathcal{I}=\int_{0}^{\infty}\left[\left(1-\frac{1}{2} e^{-\rho}\right)^{N_{f}}\right]\left(\frac{1}{\bar{\rho}} e^{-\frac{\rho}{\bar{\rho}}}\right) d \rho .
$$

By integration by parts with $u=\left(1-(1 / 2) e^{-\rho}\right)^{N_{f}}$ and $d v=$ $(1 / \bar{\rho}) e^{-\rho / \bar{\rho}} d p$, we can express $\mathcal{I}=\left[u v-\int v d u\right]_{0}^{\infty}$ as

$$
\begin{aligned}
\mathcal{I}= & {\left[\left(1-\frac{1}{2} e^{-\rho}\right)^{N_{f}}\left(-e^{-\frac{\rho}{\bar{\rho}}}\right)\right]_{0}^{\infty} } \\
& +\int_{0}^{\infty} e^{-\frac{\rho}{\bar{\rho}}} N_{f}\left(1-\frac{1}{2} e^{-\rho}\right)^{N_{f}-1}\left(\frac{1}{2} e^{-\rho}\right) d \rho \\
= & \left(\frac{1}{2}\right)^{N_{f}}+\frac{N_{f}}{2} \int_{0}^{\infty}\left(1-\frac{1}{2} e^{-\rho}\right)^{N_{f}-1} e^{-\rho\left(1+\frac{1}{\bar{\rho}}\right)} d \rho .
\end{aligned}
$$

Continuing the integration by parts with $u=(1-$ $\left.(1 / 2) e^{-\rho}\right)^{N_{f-1}}$ and $d v=e^{-\rho(1+1 / \bar{\rho})} d p$, we can write $\mathcal{I}$ as

$$
\begin{aligned}
\mathcal{I}=\left(\frac{1}{2}\right)^{N_{f}} & +\frac{N_{f}}{2}\left(\frac{1}{2}\right)^{N_{f}-1} \frac{1}{1+\frac{1}{\bar{\rho}}}+\frac{N_{f}}{2} \cdot \frac{N_{f}-1}{2} \\
& \cdot \frac{1}{1+\frac{1}{\bar{\rho}}} \int_{0}^{\infty}\left(1-\frac{1}{2} e^{-\rho}\right)^{N_{f}-2} e^{-\rho\left(2+\frac{1}{\bar{\rho}}\right)} d \rho .
\end{aligned}
$$

By performing the integration by parts of the last term and so on, after some manipulation, $\mathcal{I}$ can be written as

$$
\begin{aligned}
\mathcal{I}= & \left(\frac{1}{2}\right)^{N_{f}}+\left(\frac{1}{2}\right)^{N_{f}} \frac{N_{f}}{1+\frac{1}{\bar{\rho}}}+\left(\frac{1}{2}\right)^{N_{f}} \frac{N_{f}}{1+\frac{1}{\bar{\rho}}} \cdot \frac{N_{f}-1}{2+\frac{1}{\bar{\rho}}} \\
& +\left(\frac{1}{2}\right)^{N_{f}} \frac{N_{f}}{1+\frac{1}{\bar{\rho}}} \cdot \frac{N_{f}-1}{2+\frac{1}{\bar{\rho}}} \cdot \frac{N_{f}-2}{3+\frac{1}{\bar{\rho}}} \\
& +\ldots\left(\frac{1}{2}\right)^{N_{f}} \frac{N_{f}}{1+\frac{1}{\bar{\rho}}} \cdot \frac{N_{f}-1}{2+\frac{1}{\bar{\rho}}} \cdots \frac{2}{\left(N_{f}-1\right)+\frac{1}{\bar{\rho}}} \\
& +\left(\frac{1}{2}\right)^{N_{f}} \frac{N_{f}}{1+\frac{1}{\bar{\rho}}} \cdot \frac{N_{f}-1}{2+\frac{1}{\bar{\rho}}} \cdots \frac{2}{\left(N_{f}-1\right)+\frac{1}{\bar{\rho}}} \\
& \cdot \int_{0}^{\infty} e^{-\rho\left(N_{f}+\frac{1}{\bar{\rho}}\right)} d \rho
\end{aligned}
$$

in which the last integral $\int_{0}^{\infty} e^{-\rho\left(N_{f}+1 / \bar{\rho}\right)} d p=1 / N_{f}+1 / \bar{\rho}$. Hence, $\mathcal{I}$ can be written as

$$
\mathcal{I}=\left(\frac{1}{2}\right)^{N_{f}}\left[1+\sum_{k=1}^{N_{f}} \prod_{l=1}^{k} \frac{N_{f}+1-l}{l+\frac{1}{\bar{\rho}}}\right] .
$$

Hence, the frame error probability of DPSK under quasi-static Rayleigh-fading channels is

$$
P_{F, \text { DPSK }}=1-\left(\frac{1}{2}\right)^{N_{f}}\left[1+\sum_{k=1}^{N_{f}} \prod_{l=1}^{k} \frac{N_{f}+1-l}{l+\frac{1}{\bar{\rho}}}\right] \text {. }
$$

When $N_{f}=1$, the $P_{F, \text { DPSK }}$ becomes $1 / 2(1+\bar{\rho})$ which corresponds to the BEP of DPSK under Rayleigh-fading channels as expected.

\section{ACKNOWLEDGMENT}

The authors are grateful to the anonymous reviewers for their insightful and constructive comments.

\section{REFERENCES}

[1] S. M. Alamouti, "A simple transmitter diversity scheme for wireless communications," IEEE J. Sel. Areas Commun., vol. 16, no. 8, pp. 1451-1458, Oct. 1998.

[2] V. Tarokh and H. Jafarkhani, "A differential detection scheme for transmit diversity," IEEE J. Sel. Areas Commun., vol. 18, no. 7, pp. 1169-1174, Jul. 2000.

[3] A. Sendonaris, E. Erkip, and B. Aazhang, "User cooperation diversity-Part I: System description," IEEE Trans. Commun., vol. 51, pp. 1927-1938, Nov. 2003.

[4] J. N. Laneman, D. N. C. Tse, and G. W. Wornell, "Cooperative diversity in wireless networks: Efficient protocols and outage behavior," IEEE Trans. Inf. Theory, vol. 50, no. 12, pp. 3062-3080, Dec. 2004.

[5] J. N. Laneman and G. W. Wornell, "Distributed space-time-coded protocols for exploiting cooperative diversity in wireless networks," IEEE Trans. Inf. Theory, vol. 49, no. 10, pp. 2415-2425, Oct. 2003.

[6] T. E. Hunter and A. Nosratinia, "Cooperation diversity through coding," in Proc. IEEE Int. Symp. Inf. Theory (ISIT), 2002, p. 220.

[7] M. Janani, A. Hedayat, T. E. Hunter, and A. Nosratinia, "Coded cooperation in wireless communications: Space-time transmission and iterative decoding," IEEE Trans. Signal Process., vol. 52, no. 2, pp. 362-371, Feb. 2004.

[8] P. A. Anghel, G. Leus, and M. Kaveh, "Multi-user space-time coding in cooperative networks," in Proc. IEEE Int. Conf. Acoust., Speech, Signal Process., May 2003, pp. IV73-IV76.

[9] I. Hammerstroem, M. Kuhn, B. Rankov, and A. Wittneben, "Space-time processing for cooperative relay networks," in Proc. IEEE Fall Veh. Technol. Conf., Oct. 2003, pp. 404-408.

[10] R. U. Nabar and H. Bölcskei, "Space-time signal design for fading relay channels," in Proc. IEEE GLOBECOM, Dec. 2003, pp. 1952-1956.

[11] C. Gao and A. M. Haimovich, "BER analysis of MPSK space-time block codes with differential detection," IEEE Commun. Lett., vol. 7, no. 7, pp. 314-316, Jul. 2003.

[12] J. G. Proakis, Digital Communications, 3rd ed. New York: McGrawHill, 1995.

[13] R. U. Nabar, H. Bölcskei, and F. W. Kneubühler, "Fading relay channels: performance limits and space-time signal design," IEEE J. Sel. Areas Commun., vol. 22, no. 6, pp. 1099-1109, Aug. 2004.

[14] D. Chen and J. N. Laneman, "Noncoherent demodulation for cooperative diversity in wireless systems," in Proc. IEEE GLOBECOM, Dec. 2004, pp. 31-35.

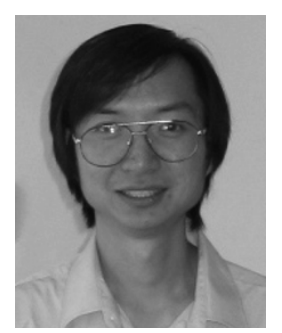

Poramate Tarasak (S'97-M'05) received the B.Eng. degree in electrical engineering from Chulalongkorn University, Bangkok, Thailand, in 1997, the M.Eng. degree in telecommunications from the Asian Institute of Technology (AIT), Pathumthani, Thailand, in 1999, and the Ph.D. degree in electrical engineering from the University of Victoria, Victoria, BC, Canada, in 2004.

Since October 2004, he has been a BK Postdoctoral Fellow at the Department of Electrical Engineering, Korea Advanced Institute of Science and Technology (KAIST), Daejeon, Korea. His current research interest is cooperative diversity. 


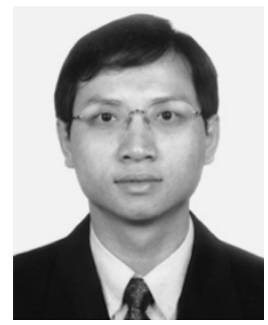

Hlaing Minn (S'99-M'01) received the B.E. degree in electronics from the Yangon Institute of Technology, Yangon, Myanmar, in 1995, the M.Eng. degree in telecommunications from the Asian Institute of Technology (AIT), Pathumthani, Thailand, in 1997, and the Ph.D. degree in electrical engineering from the University of Victoria, Victoria, BC, Canada, in 2001.

He was with the Telecommunications Program, AIT, as a Laboratory Supervisor during 1998. He was a Research Assistant from 1999 to 2001, and a Postdoctoral Research Fellow during 2002 in the Department of Electrical and Computer Engineering, University of Victoria. Since September 2002, he has been with the Erik Jonsson School of Engineering and Computer Science, University of Texas at Dallas, as an Assistant Professor. His research interests include wireless communications, statistical signal processing, error control, detection, estimation, synchronization, signal design, and cross-layer design.

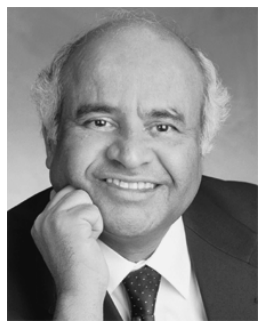

Vijay K. Bhargava (S'70-M'74-SM'82-F'92) received the B.Sc., M.Sc., and Ph.D. degrees from Queen's University, Kingston, ON, Canada in 1970, 1972, and 1974 respectively.

Currently, he is a Professor and Head of the Department of Electrical and Computer Engineering, University of British Columbia, Vancouver, BC, Canada. Previously, he was with the University of Victoria (1984-2003) and with Concordia University, Montréal (1976-1984). He is coauthor of Digital Communications by Satellite (New York: Wiley, 1981), coeditor of Reed-Solomon Codes and Their Applications (New York: IEEE, 1994), and coeditor of Communications, Information and Network Security (Norwell, MA: Kluwer, 2003). His research interests are in wireless communications.

Dr. Bhargava is a Fellow of the Engineering Institute of Canada (EIC), the Canadian Academy of Engineering, and the Royal Society of Canada. He is a recipient of the IEEE Centennial Medal (1984), the IEEE Canada's McNaughton Gold Medal (1995), the IEEE Haraden Pratt Award (1999), the IEEE Third Millennium Medal (2000), the IEEE Graduate Teaching Award (2002), and the Eadie Medal of the Royal Society of Canada (2004). He is very active in the IEEE and was nominated by the IEEE Board of Director for the Office of IEEE President-Elect. He has served on the Board of the IEEE Information Theory Society and the IEEE Communications Society. He is a Past President of the IEEE Information Theory Society. He is an Editor for the IEEE TRANSACTIONS ON WIRELESS COMMUNICATIONS. 